Proceedings

\title{
Screen-Printed Microcantilevers for Environmental Sensing ${ }^{\dagger}$
}

\author{
Simon Grall ${ }^{1,2}$, Hélène Debéda ${ }^{1, *}$, Isabelle Dufour ${ }^{1}$ and Vincent Aubry ${ }^{2}$ \\ 1 Laboratoire IMS, Université de Bordeaux, 33400 Talence, France; simon.grall@mail.com (S.G.); \\ isabelle.dufour@ims-bordeaux.fr (I.D.) \\ 2 Groupe PSA, 78140 Vélizy-Villacoublay, France; vincent.aubry@mpsa.com \\ * Correspondence: helene.debeda@ims-bordeaux.fr \\ † Presented at the Eurosensors 2018 Conference, Graz, Austria, 9-12 September 2018. \\ Published: 16 July 2019
}

\begin{abstract}
This work investigates different geometries for fully screen-printed self-actuated selfreadout microcantilevers made using a sacrificial layer process. The microcantilevers are made of PZT sandwiched between two gold electrodes. Q-factors as high as 1300 are reported. Promising theoretical sensitivities are reported $(1000 \mathrm{~Hz} / \mu \mathrm{g}$ at $620 \mathrm{kHz})$ and applications as humidity, temperature and particles sensor are explored in this work.
\end{abstract}

Keywords: piezoelectric; PZT; micro-resonator; airborne particles

\section{Introduction}

More and more regions of the world are facing the challenges of air pollution due to gases and fine particulate matters (PM). This is especially true in fast-developing countries with rampant industrialisation releasing massive amount of dusts in the atmosphere. Recent reports of the WHO recommend for PM of $10 \mu \mathrm{m}$ and $2.5 \mu \mathrm{m}$ diameters a maximal exposure over $24 \mathrm{~h}$ of $50 \mu \mathrm{g} / \mathrm{m}^{3}$ and 25 $\mu \mathrm{g} / \mathrm{m}^{3}$ respectively [1], which gives guidelines regarding the sensitivities to be achieved with PM sensors. Silicon microresonators have been widely used as sensors, including for particles detection $[2,3]$. In this work we propose self-actuated self-readout piezoelectric microcantilevers used as a gravimetric sensor in the scope of particulate matter detection for automotive application. It has already been demonstrated that such microcantilevers can be used as specific gas sensors in previous work [4]. Applications as a temperature and humidity sensor are explored. A test bench is mounted for particulate matter sensing. First results showed the proof of concept and further experiments are currently running with promising perspectives.

\section{Materials and Methods}

The microcantilevers are screen-printed using a sacrificial layer process [5], further described in the Appendix A. Made of a $\mathrm{PbZr}_{0.52} \mathrm{Ti}_{0.48} \mathrm{O}_{3}$ (PZT) ceramic beam sandwiched between two gold electrodes (Figure 1), the clamped-free microcantilevers are entirely screen-printed on alumina substrates. They are sintered at $900{ }^{\circ} \mathrm{C}$ where the sacrificial layer burns, releasing the beam during the sintering of the ceramics. High densification (88\% of the bulk PZT) and high Q-factors (1300) are achieved. The unusual 31-longitudinal resonance mode is here privileged to obtain high resonant frequencies. Different geometries are investigated from $8 \times 2 \times 0.1 \mathrm{~mm}^{3}$ down to $1 \times 1 \times 0.1 \mathrm{~mm}^{3}$. The resonant frequency of the microcantilever is measured using a network analyser. The resonance frequency $f_{0}$ is followed through the maximum of the conductance $G$, image of the mechanical resonance. A polynomial model (Equation (1)) allows precise determination of the resonant frequency through the conductance measurement (Figure 2). Details about this equation are given in 
Appendix B. This is consistent with the low noise $(\sigma)$ reported along the whole range of geometries investigated. Theoretical sensitivities are calculated and showed up to $1000 \mathrm{~Hz} / \mu \mathrm{g}$ at $620 \mathrm{kHz}$ (Table 1).

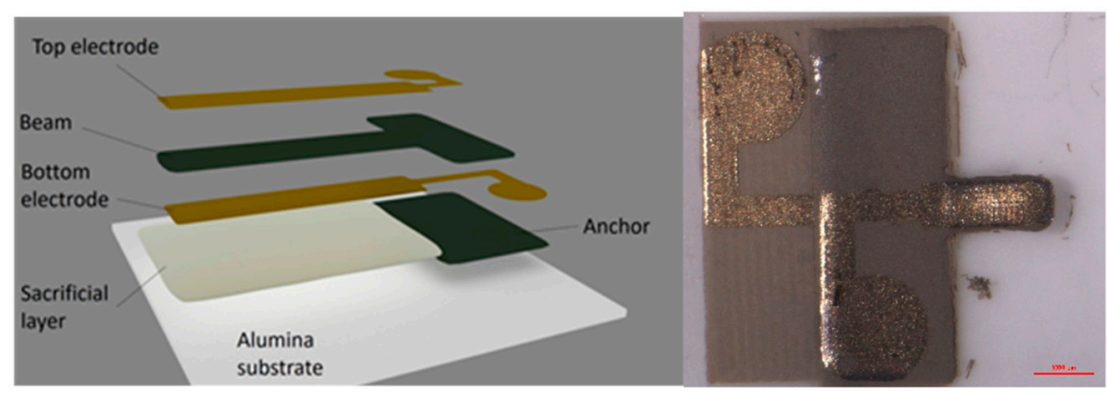

(a)

(b)

Figure 1. (a) Exploded view of the layer piling. (b) Photo of a $2 \times 1 \times 0.1 \mathrm{~mm}^{3}$ microcantilever.

$$
\left.G \approx 2 \pi C Q f_{0}\left(1-4 Q^{2}+8 Q^{2} \frac{f}{f_{0}}-4 Q^{2} \frac{f^{2}}{f_{0}^{2}}\right)+\cdots\right)
$$

This expression is used to fit the raw data and allows a more precise determination of the resonant frequency as illustrated in Figure 2.

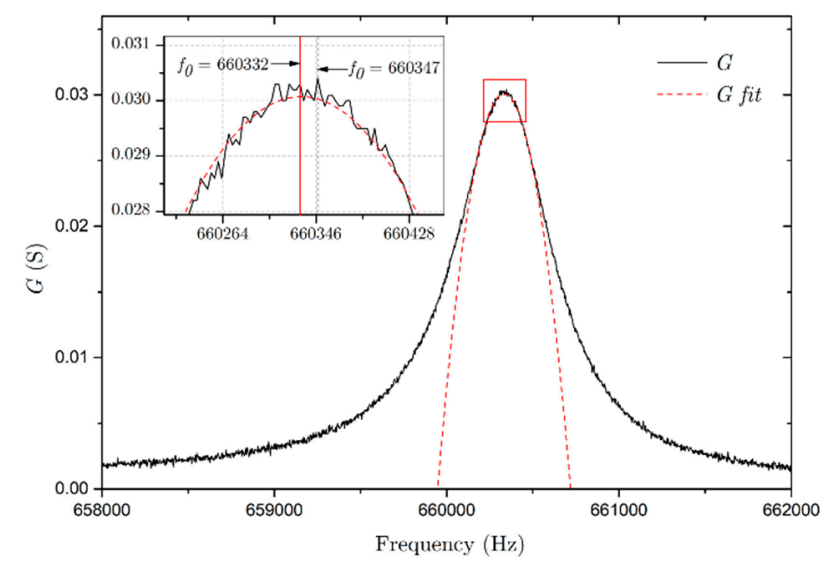

Figure 2. Conductance peak $(Q=1300)$ at the resonance with polynomial model of $G$ for resonant frequency determination.

A test bench is mounted for dust detection experiments, represented Figure 3. An optical particle analyser (Fidas ${ }^{\circledR}$ Frog, Palas Gmbh, Karlsruhe, Germany) put at the end of the gas line is used as reference. The microcantilever is actuated beforehand for 20 min under a clean nitrogen flow (1 $\mathrm{L} / \mathrm{min}$ ) for stabilisation of humidity and temperature. For temperature and humidity measurements, the microcantilevers are put in a climatic chamber in which humidity and temperature can be controlled. An additional temperature and humidity sensor is added in the chamber as close as possible from the microcantilever. Twenty minutes-plateaus of humidity are carried out from $50 \%$ to $90 \%$ by steps of $10 \%$ of relative humidity (\%RH), followed by temperature plateaus ranging from 25 ${ }^{\circ} \mathrm{C}$ to $45^{\circ} \mathrm{C}$ by steps of $5{ }^{\circ} \mathrm{C}$. 


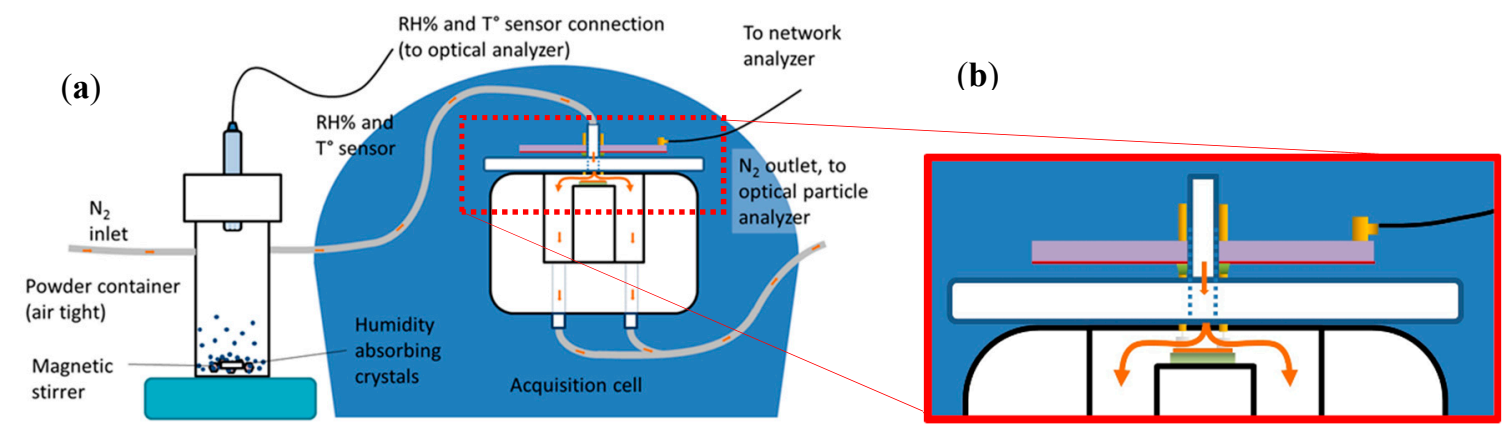

Figure 3. (a) Scheme of the test bench and (b) zoom on the microcantilever, whose largest area is perpendicular to the flow. Polystyrene powder (mean diameter $=2.5 \mu \mathrm{m}$ ) is used as airborne particles.

Table 1. Theoretical ( $f_{0}$ th.) and experimental ( $f_{0}$ exp.) resonant frequencies, theoretical sensitivities $(S)$, noise $\sigma$ and quality factor $Q$ exp.. The noise $\sigma$ and the quality factor $Q$ exp. are measured on one microcantilever for each dimension. $f_{0}$ exp. is the mean value obtained for each geometry. $S$ calculations are made using $f_{0}$ exp. values. Refer to Appendix B for more details.

\begin{tabular}{cccccc}
\hline $\mathbf{L} / \mathbf{w}(\mathbf{m m}) \boldsymbol{f}_{\mathbf{0}}$ th. $\mathbf{( k H z )} \boldsymbol{f}_{\mathbf{0}}$ exp. $(\mathbf{k H z}) \boldsymbol{\sigma}(\mathbf{H z}) \boldsymbol{Q}$ exp.S $\mathbf{( H z / \mu g )}$ \\
\hline $8 / 2$ & 90 & 90 & 1.5 & 100 & -5 \\
$8 / 1$ & 90 & 100 & 3.6 & 150 & -10 \\
$4 / 2$ & 170 & 180 & 0.9 & 250 & -20 \\
$4 / 1$ & 170 & 180 & 0.9 & 300 & -40 \\
$2 / 2$ & 370 & 340 & 0.8 & 250 & -100 \\
$2 / 1$ & 360 & 340 & 0.7 & 600 & -200 \\
$1 / 2$ & 810 & 780 & 1.2 & 800 & -400 \\
$1 / 1$ & 790 & 620 & 0.6 & 1300 & -1000 \\
\hline
\end{tabular}

\section{Results and Discussion}

Humidity and temperature are steady during the dust experiments. The measurements are carried out on a $1 \times 1 \times 0.1 \mathrm{~mm}^{3}$ microcantilever. On the Figure 4 , the first part of the curves shows a slowly increasing of $f_{0}$, corresponding to a mechanical stabilisation of the resonance. Then as particles are released, $f_{0}$ decreased constantly, resulting in a total shift of $23 \mathrm{~Hz}$. Considering the theoretical sensitivity for this specific device, the mass of deposited particles can be estimated around $23 \mathrm{ng}$, giving collection efficiency around $12 \mathrm{ppm}$ at $1 \mathrm{~L} / \mathrm{min}$. Experiments in the climatic chamber show that the resonant frequency of the microcantilever varies negatively with both temperature and humidity, with respectively $-47 \mathrm{~Hz} /{ }^{\circ} \mathrm{C}$ and $-41 \mathrm{~Hz} / \% \mathrm{RH}$ for a $1 \times 1 \times 0.1 \mathrm{~mm}^{3}$ microcantilever (Figure 5). For the temperature, this is most likely due to changes in the stiffness of the beam, and for the humidity to the mass effect of the adsorbed water on the microcantilever. The wavy shape of the temperature profile is due to small spikes of humidity during temperature steps, both not being perfectly independent in the chamber. 


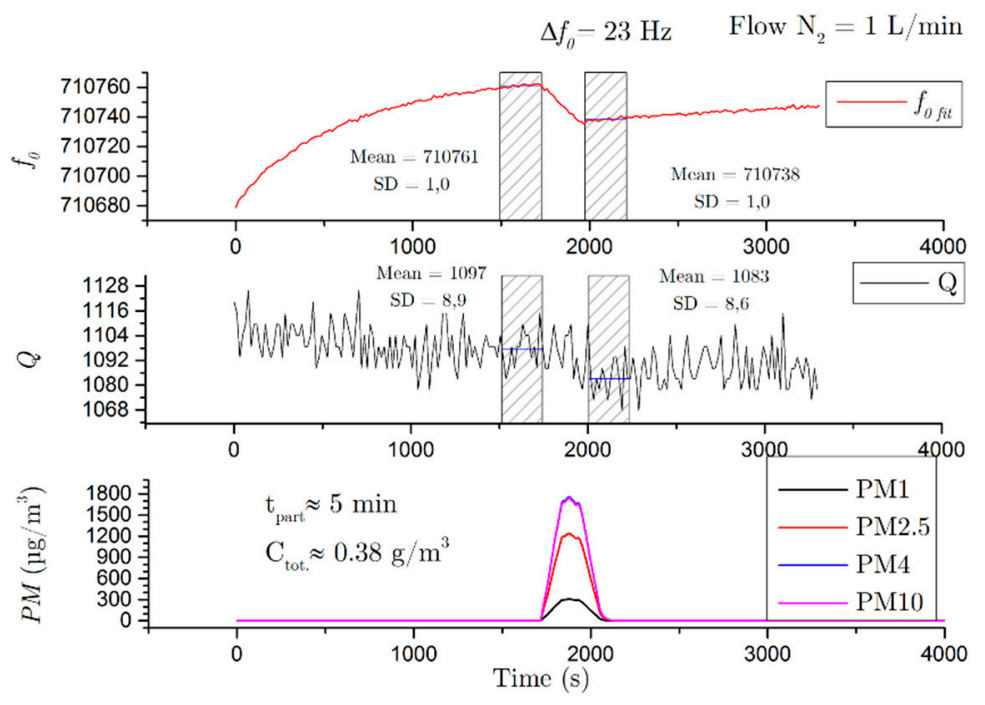

Figure 4. Resonant frequency $f_{0}$, quality factor $Q$ and $P M$ evolution over time. tpart. is the duration of the dust exposure, and Ctot. the total of particles that transited in front of the microcantilever sensor. Mean value and standard deviation (SD) are indicated before and after dust input.

(a)

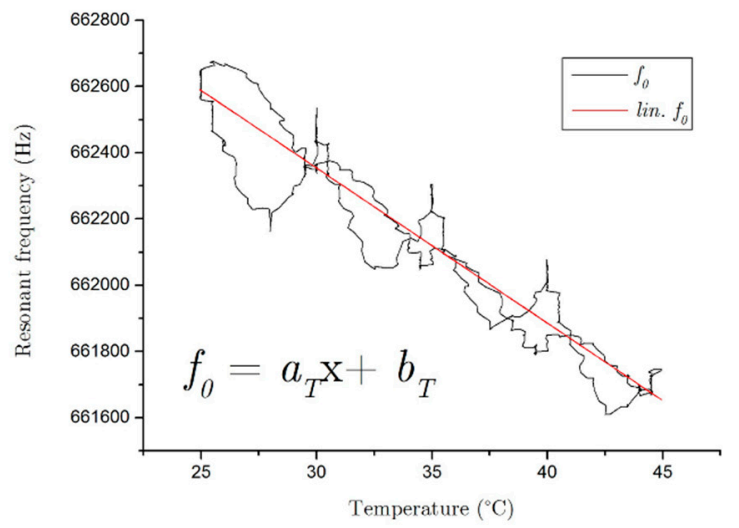

(b)

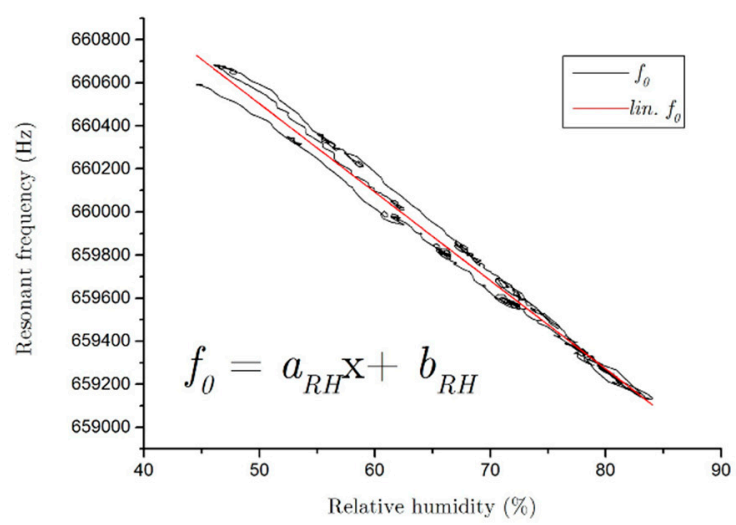

Figure 5. Impact of the temperature (a) and humidity (b) on the resonant frequency $f_{0}$ of a $1 \times 1 \times 0.1 \mathrm{~mm}^{3}$ microcantilever, with $a T=-47 \mathrm{~Hz} /{ }^{\circ} \mathrm{C}, b T=663,759 \mathrm{~Hz}, a R H=-41 \mathrm{~Hz} / \% \mathrm{RH}$ and $b R H=662,557 \mathrm{~Hz}$.

\section{Conclusions}

This work showed proof of concept for screen-printed microcantilevers to be used as fine particles sensors. This sensor can also be used either as a temperature or humidity sensor with high sensitivities. Other experiments are currently running toward improving the particles collection efficiency using thermophoresis and decreasing the dust carrying flow as already explored by Liu et al. for SAW devices [6]. As new materials are being investigated worldwide, other improvements could also be made in using more ecological alternatives to PZT such as BNT or KNN piezoceramics [7].

Author Contributions: S.G., H.D. conceived, designed and made the microcantilevers, S.G. conceived, designed and performed the dust, humidity and temperature experiments, S.G., H.D. and I.D. analyzed the data; S.G., H.D., I.D. and V.A. wrote the paper.

Funding: Peugeot Citroën Automobiles and ANRT (Association Nationale de la Recherche et de la Technologie)

Acknowledgments: The authors want to thank the OpenLab structure between Groupe PSA and Laboratoire IMS for supporting this work. 
Conflicts of Interest: The authors declare no conflict of interest.

\section{Appendix A. Fabrication: The Microcantilevers are Entirely Screen-Printed in the Order Indicated Table A1.}

Table A1. Screen-printing steps and materials used for the layers. For gold and sacrificial layer, ESL8836 and ESL244t commercial inks were used respectively. The PZT ink is homemade.

\begin{tabular}{cccc}
\hline Step & Layer & \multicolumn{2}{c}{ Material } \\
\hline 1 & Anchor & PZT & 35 \\
2 & Sacrificial Layer & Polyester & 35 \\
3 & Bottom Electrode & Gold & 15 \\
4 & Beam & PZT & 120 \\
5 & Top Electrode & Gold & 8 \\
\hline
\end{tabular}

${ }^{1}$ Thickness indicated after drying and before firing of the layers.

All samples are dried/cross-linked at $120^{\circ} \mathrm{C}$ between each step. The devices are then pressed isostatically at $40 \mathrm{MPa}$ is applied at $65{ }^{\circ} \mathrm{C}$ for $4 \mathrm{~min}$ and fired at $900{ }^{\circ} \mathrm{C}$ for $2 \mathrm{~h}$. More details on the fabrication process are available in an article to be published.

\section{Appendix B. Equations}

The polynomial expression of $G$ is derived from the Equation (B1), which is the conductance the equivalent circuit of a piezoelectric material sandwiched between two conductive layers $\left(R, L, C / / C_{0}\right)$, neglecting leaking current. In this equation, $Q$ is the quality factor, $f$ the frequency, $f_{0}$ the resonant frequency, $C$ the capacitance of the motional branch.

$$
G(f)=\frac{\frac{2 \pi C f^{2}}{Q f_{0}}}{1+\left(\frac{1}{Q^{2}}-2\right) \frac{f^{2}}{f_{0}^{2}}+\frac{f^{4}}{f_{0}^{4}}}
$$

Using Taylor expansions leads to the Equation (1) in the main text.

The Table 1 in the main text is obtained using the following equations:

$$
f_{0}^{i}=\frac{(2 i-1)}{4 L} \sqrt{\frac{E_{P Z T} h_{P Z T}+2 E_{\text {gold }} h_{\text {gold }}}{\rho_{P Z T} h_{P Z T}+2 \rho_{\text {gold }} h_{\text {gold }}}}
$$

in which $E_{X}, p_{X}$ and $\boldsymbol{h}_{X}$ are respectively the Young's modulus, the density and the thickness of the layer $X$, and $L$ the length of the cantilever,

$$
S=\frac{-f_{0}}{2 m}
$$

in which $m$ is the mass of the cantilever.

The Equation (B2) gives the resonant frequency in the 3-1 longitudinal mode at the $i^{\text {th }}$ order. In the table, it is used to fill the column $f_{0}$ th.

\section{References}

1. World Health Organization. Evolution of WHO Air Quality Guidelines-Past, Present and Future. 2017; p. 21. (Available online: http://www.euro.who.int (accessed on 6 December 2018).

2. Boisen, A.; Dohn, S.; Keller, S.S.; Schmid, S.; Tenje, M. Cantilever-like micromechanical sensors. Rep. Prog. Phys. 2011, 74, 36101, doi:10.1088/0034-4885/74/3/036101.

3. Wasisto, H.S.; Uhde, E.; Peiner, E. Enhanced performance of pocket-sized nanoparticle exposure monitor for healthy indoor environment. Build. Environ. 2016, 95, 13-20, doi:10.1016/j.buildenv.2015.09.013. 
4. Clément, P.; Perez, E.D.; Gonzalez, O.; Calavia, R.; Lucat, C.; Llobet, E.; Debéda, H. Gas discrimination using screen-printed piezoelectric cantilevers coated with carbon nanotubes. Sens. Actuator, B Chem. 2016, 237, 1056-1065, doi:10.1016/j.snb.2016.07.163.

5. Debéda, H.; Lucat, C.; Pommier-Budinger, V. Printed Piezoelectric Materials for Vibration-based Damage Detection. Procedia Eng. 2016, 168, 708-712, doi:10.1016/j.proeng.2016.11.254.

6. Liu, J.; Hao, W.; Liu, M.; Liang, Y.; He, S. A Novel Particulate Matter 2.5 Sensor Based on Surface Acoustic Wave Technology. Appl. Sci. 2018, 8, 82, doi:10.3390/app8010082.

7. Hong, C.H.; Kim, H.P.; Choi, B.Y.; Han, H.S.; Son, J.S.; Ahn, C.W.; Jo, W. Lead-free piezoceramics-Where to move on? J. Mater. 2016, 2, 1-24, doi:10.1016/j.jmat.2015.12.002.

(C) 2018 by the authors. Licensee MDPI, Basel, Switzerland. This article is an open access article distributed under the terms and conditions of the Creative Commons Attribution (CC BY) license (http://creativecommons.org/licenses/by/4.0/). 